\title{
Spontaneous tensorization from curvature coupling and beyond
}

\author{
Fethi M. Ramazanoğlu ${ }^{1}$ \\ ${ }^{1}$ Department of Physics, Koç University, \\ Rumelifeneri Yolu, 34450 Sariyer, Istanbul, Turkey
}

(Dated: April 16, 2019)

\begin{abstract}
We generalize the recently introduced extended scalar-tensor-Gauss-Bonnet (ESTGB) theories and their close relatives to include spontaneous growth of nonscalar fields such as vectors. This is analogous to the program that developed spontaneous tensorization from the original spontaneous scalarization theory of Damour and Esposito-Farèse (DEF). The new larger family of theories conserves the appeal of the DEF theory in terms of conforming to weak-field tests and also providing large signals in strong gravity. Moreover, they provide a much richer phenomenology including spontaneous tensorization of black holes as in ESTGB. These theories, together with other possible future extensions that we discuss, testify to the ubiquity of spontaneous growth in gravity. We also note that theories with derivative coupling require special attention since they can lead to potentially problematic higher derivatives in the equations of motion.
\end{abstract}

PACS numbers:

\section{INTRODUCTION}

The age of gravitational waves has provided new opportunities to test gravity in the strong and dynamical field regime, which in turn has been driving many efforts to understand possible deviations from general relativity (GR) 1, 2. One of the leading avenues in this area has been the investigation of spontaneous scalarization. This phenomenon was originally proposed by Damour and Esposito-Farèse (DEF) in scalar-tensor theories, where the matter coupling of the scalar leads to an effective scalar mass term with the "wrong" sign [3]. The resulting tachyonic instability of the GR solution in the presence of matter causes astrophysical neutron stars to have stable scalar clouds around them.

The main appeal of spontaneous scalarization is twofold. First, the scalar dies off fast away from the star, and satisfies the weak-field tests that strongly disfavor other variants of scalar-tensor theories 4. Second, deviations from GR are large near a neutron star, and hence are easier to detect with gravitational wave observations which currently have relatively low precision [5]. These factors have inspired the investigation of the spontaneous scalarization phenomena in theories beyond their original context. A recent development in this regard is the extended scalar-tensor-Gauss-Bonnet (ESTGB) theories where the scalar couples to the Gauss-Bonnet term rather than matter [6] 8 . The mechanism works similarly, where the tachyonic instability is incited by curvature rather than matter, and hence can lead to scalarized black holes as well as neutron stars. The idea can also be extended to other higher-curvature terms, or to more generic terms such as an Einstein-Maxwell-scalar action in a relatively straightforward manner 9 .

A second program to generalize spontaneous scalarization has been undertaken in parallel to ESTGB theories, where there is still matter coupling as in the DEF theory, but the scalar is replaced by some other field, e.g. a vector, or the nature of the coupling is modified to in- clude derivative terms. The former has led to theories of spontaneous vectorization [10, and the latter to spontaneous scalarization based on ghostlike instabilities rather than tachyons [11. These general phenomena of spontaneous growth are categorized under the umbrella term "spontaneous tensorization."

The main theme of the current study is to combine the two aforementioned avenues to generalize spontaneous scalarization. We consider fields that couple to a general action term such as the Gauss-Bonnet term (instead of just matter). However, we allow the fields to be nonscalars, e.g. vectors, or we allow derivative couplings. Such fields can also go through spontaneous growth with suitable coupling functions which lead to a further generalization of spontaneous scalarization we call "extended spontaneous tensorization." These novel theories preserve the appeal of the DEF theory in weak and strong gravity regimes while providing a richer phenomenology. However, they may also feature higher-derivative terms in the field equations in cases with derivative couplings, which does not occur for derivative coupling to matter. We will employ $G=c=1$ throughout the paper.

\section{SPONTANEOUS SCALARIZATION IN EXTENDED SCALAR-TENSOR THEORIES}

Let us first review the known generalizations of spontaneous scalarization starting with ESTGB theory for which the coupling of the Gauss-Bonnet term in the action depends on a dynamical scalar field $[6]]^{1}$

$$
\frac{1}{16 \pi} \int d^{4} x \sqrt{-g}\left[R-2 \nabla_{\mu} \phi \nabla^{\mu} \phi+\lambda^{2} f(\phi) \mathcal{R}^{2}\right],
$$

\footnotetext{
1 This idea was proposed by three different groups, but our discussion is closer to Refs. 6] 7] which emphasized the role of instabilities and spontaneous scalarization.
} 
where $\mathcal{R}^{2}=R^{2}-4 R_{\mu \nu} R^{\mu \nu}+R_{\mu \nu \rho \sigma} R^{\mu \nu \rho \sigma}$ is the GaussBonnet invariant, $\phi$ is a dynamical scalar field with curvature coupling $f(\phi)$, and $\lambda$ is a constant whose dimension ensures that $f(\phi)$ is dimensionless. The most relevant equation of motion for our discussion is that of the scalar

$$
\square \phi=-\frac{\lambda^{2}}{4} f^{\prime}(\phi) \mathcal{R}^{2}
$$

We will use ' to denote the derivative of any function with respect to its argument, in this case $\phi$.

Assume that the first derivative of $f$ vanishes at $\phi=0$, and consider the linearization of Eq. 2

$$
\square \phi \approx-\frac{\lambda^{2}}{4} \widehat{f^{\prime \prime}(\phi)} \mathcal{R}^{2} \phi \equiv m_{\mathrm{eff}}^{2} \phi,
$$

where an overhat denotes the value of a function when its argument (in this case $\phi$ ) is 0 . This is the wave equation for a scalar field with effective mass $m_{\text {eff }}$, even though we have a massless scalar in the action. The case of interest to us is when $f^{\prime \prime}(\phi)>0$ and $m_{\text {eff }}$ is imaginary, i.e. $\phi$ is a tachyon. A Fourier mode $e^{i(\vec{k} \cdot \vec{x}-\omega t)}$ with $\omega \sim$ $\pm \sqrt{k^{2}+m_{\text {eff }}^{2}}$ would undergo exponential growth rather than oscillations for small $k$. In other words, there is an infrared instability around $\phi=0$, but depending on the form of $f(\phi)$, nonlinear terms can eventually stop this growth leading to a stable scalar field solution [12 14. Such solutions were explicitly constructed for spherically symmetric black holes and neutron stars, circumventing no-hair results 6 6].

A standard function that provides all of these features is $f(\phi)=1-e^{\beta \phi^{2} / 2}$ for some negative constant $\beta$ [7], but any function with similar parabolic behavior around $\phi=0$ and decaying derivatives at large scalar values provides qualitatively similar results. The decay diminishes the effective mass term with growing scalar field values, preventing a runaway instability.

One can immediately see that the specific form of the Gauss-Bonnet term does not play a specific role in Eqs. 1. 3. so replacing it with another nonvanishing action term still leads to spontaneous scalarization. The action for the most general such theory is

$$
\frac{1}{16 \pi} \int d^{4} x \sqrt{-g}\left[R-2 \nabla_{\mu} \phi \nabla^{\mu} \phi+\bar{\lambda}^{2} f(\phi) \mathcal{L}_{\chi}\left[\chi, g_{\mu \nu}\right]\right]
$$

with linearized scalar equation of motion

$$
\square \phi \approx-\frac{\bar{\lambda}^{2}}{4} \mathcal{L}_{\chi} \widehat{f^{\prime \prime}(\phi)} \phi \equiv m_{\mathrm{eff}}^{2} \phi
$$

if $\widehat{f^{\prime}(\phi)}=0 . \quad \mathcal{L}_{\chi}$ can be a purely metric term as in Gauss-Bonnet or Chern-Simons gravity [15, or depend on another field $\chi$ such as the Maxwell action of a vector field $\mathcal{L}_{\chi}=F_{\mu \nu} F^{\mu \nu}$ [9]. We will call this family of theories with generic $\mathcal{L}_{\chi}$ "extended spontaneous scalarization theories".
Hairy compact object solutions are not a complete novelty, and the case of neutron stars has been known since the scalar-tensor theories of Damour and EspositoFarèse [3. Consider the action

$$
\begin{gathered}
\frac{1}{16 \pi} \int d^{4} x \sqrt{-g}\left[R-2 \nabla_{\mu} \phi \nabla^{\mu} \phi\right] \\
+S_{m}\left[\psi_{m}, A^{2}(\phi) g_{\mu \nu}\right]
\end{gathered}
$$

where $S_{m}$ is the action for any matter field $\psi_{m}$, with the peculiarity that matter does not couple to $g_{\mu \nu}$ (minimal coupling), but to a conformally scaled metric $\tilde{g}_{\mu \nu}=$ $A^{2} g_{\mu \nu}$. This gives the scalar equation of motion

$$
\square \phi=-8 \pi \tilde{T} A^{3} \frac{\widehat{d A}}{d\left(\phi^{2}\right)} \phi
$$

where $\tilde{T}$ is the trace of the matter stress-energy tensor with respect to $\tilde{g}_{\mu \nu}$. The right-hand side behaves exactly as an effective mass term for appropriate couplings such as $A=e^{\beta \phi^{2} / 2}$ with negative $\beta$. It has been known that neutron stars scalarize in this theory in a natural part of the parameter space. This choice of $A$ also ensures that the instability gets weaker as the scalar field grows, leading to stable scalar clouds around neutron stars.

The extended spontaneous scalarization in Eq. 4 uses the same underlying mechanism as Eq. 6. effective mass generation through coupling to other fields. Despite the similarity between the two, there is much richer phenomenology in the extended case. For example, a scalar coupling to higher-derivative terms allows hairy black holes whereas spontaneous scalarization in Eq. 7 only scalarizes matter fields.

As a last note, we should add that spontaneous scalarization can occur for self-interacting scalar fields as well. The simplest case would be a massive scalar $\nabla_{\mu} \phi \nabla^{\mu} \phi \rightarrow$ $\nabla_{\mu} \phi \nabla^{\mu} \phi+m_{\phi}^{2} \phi^{2}$. The intrinsic mass term $m_{\phi}$ suppresses spontaneous growth through its positive contribution to $m_{\text {eff }}^{2}$, but one can still obtain imaginary effective mass for similar choices of $f(\phi)$. Despite this negative aspect, a massive scalar dies off much faster in the far field, providing better agreement with observations. This has been valuable for the DEF theory [16], and might be useful in extended spontaneous scalarization theories as well. For this reason, we will consider the more general massive fields in the following discussion.

\section{EXTENDED VECTOR-TENSOR THEORIES}

We have seen that the term that the scalar field couples to is not crucial for spontaneous growth. A close examination shows that the type of field that grows spontaneously is not essential either. The latter idea has already been applied to the DEF theory where one replaces the 
scalar in Eq. 6 with a vector field $X_{\mu}$,

$$
\begin{aligned}
& \frac{1}{16 \pi} \int d^{4} x \sqrt{-g}\left[R-F_{\mu \nu} F^{\mu \nu}-2 m_{X}^{2} X_{\mu} X^{\mu}\right] \\
& \quad+S_{m}\left[\psi, A_{X}^{2}(x) g_{\mu \nu}\right]
\end{aligned}
$$

where $F_{\mu \nu}=\nabla_{\mu} X_{\nu}-\nabla_{\nu} X_{\mu}$ and $x=g^{\mu \nu} X_{\mu} X_{\nu}$. We use a massive vector (Proca) field as we explained at the end of the previous section. The vector field equation of motion is

$$
\nabla_{\rho} F^{\rho \mu}=\left(-8 \pi A_{X}^{4} \Lambda \tilde{T}+m_{X}^{2}\right) X^{\mu},
$$

and the right-hand side behaves as an effective mass term in the Proca equation. An appropriate choice of $A_{X}$ such as $e^{\beta_{X} X_{\mu} X^{\mu}}$ renders the left-hand side negative, and leads to spontaneous growth of $X_{\mu}$. This phenomenon is called "spontaneous vectorization" 10. The term that covers all spontaneously growing fields is "spontaneous tensorization".

The two ideas that generalize spontaneous scalarization in scalar-tensor theories can be combined in an "extended vector-tensor-Gauss-Bonnet (EVTGB) theory"

$$
\begin{gathered}
\frac{1}{16 \pi} \int d^{4} x \sqrt{-g}\left[R-F_{\mu \nu} F^{\mu \nu}-2 m_{X}^{2} X_{\mu} X^{\mu}\right. \\
\left.+\lambda_{X}^{2} f_{X}(x) \mathcal{R}^{2}\right]
\end{gathered}
$$

with the vector equation of motion

$$
\nabla_{\rho} F^{\rho \mu}=\left(-\frac{\lambda_{X}^{2}}{2} \mathcal{R}^{2} f_{X}^{\prime}(x)+m_{X}^{2}\right) X^{\mu} .
$$

An appropriate choice of $f_{X}$ such as $f_{X}=1-e^{\beta_{X} X_{\mu} X^{\mu}} / 2$ would give $X_{\mu}$ an imaginary effective mass, leading to vectorized black holes and neutron stars. This can be immediately generalized to

$$
\begin{aligned}
\frac{1}{16 \pi} \int d^{4} x \sqrt{-g} & {\left[R-F_{\mu \nu} F^{\mu \nu}-2 m_{X}^{2} X_{\mu} X^{\mu}\right.} \\
& \left.+\bar{\lambda}_{X}^{2} f_{X}(x) \mathcal{L}_{\chi}\left[\chi, g_{\mu \nu}\right]\right]
\end{aligned}
$$

just as in the case of scalar fields in Eq. 4, with the equation of motion

$$
\nabla_{\rho} F^{\rho \mu}=\left(-\frac{\bar{\lambda}_{X}^{2}}{2} \mathcal{L}_{\chi} f_{X}^{\prime}(x)+m_{X}^{2}\right) X^{\mu} .
$$

We will call this mechanism "extended spontaneous vectorization." Similarly, the general name that covers all fields is "extended spontaneous tensorization". We note that we do not claim that $X_{\mu}$ is any known part of the Standard Model, in parallel with the case of $\phi$ in spontaneous scalarization which is understood as a hitherto unobserved fundamental field.

\section{EXTENDED SPONTANEOUS TENSORIZATION WITH GHOSTLIKE INSTABILITIES}

Extension of spontaneous growth from scalars to vectors is relatively straightforward, i.e., One changes the canonical scalar field action for the canonical vector field action, and chooses a coupling function $f$ that provides an imaginary effective mass. A vector is no more special than a scalar, and hence spontaneous growth seems to be extendable to other fields as well. However, we will first discuss another path to generalize spontaneous scalarization: using a ghost instead of a tachyon to incite an instability. This idea is again analogous to existing ideas in gravity with nonminimal matter couplings (similar to the DEF theory), but we will see that adapting such mechanisms to curvature couplings is not straightforward, and can introduce new challenges.

\section{A. Ghosts in spontaneous growth}

Changing the field that spontaneously scalarizes is not the only way to generalize spontaneous growth beyond extended scalar-tensor theories. The growth needs an instability, but this does not have to be a tachyon which has been the exclusive case so far in our discussion. In principle, a ghostlike behavior can provide the instability as well. Let us demonstrate this on a modification of the massive DEF theory

$$
\begin{aligned}
& \frac{1}{16 \pi} \int d^{4} x \sqrt{-g}\left[R-2 \nabla_{\mu} \phi \nabla^{\mu} \phi-2 m_{\phi} \phi^{2}\right] \\
& \quad+S_{m}\left[\psi, A_{\partial}^{2}(K) g_{\mu \nu}\right], K=g^{\mu \nu} \partial_{\mu} \phi \partial_{\nu} \phi .
\end{aligned}
$$

with the equation of motion

$$
\nabla_{\mu}\left[\left(-8 \pi \tilde{T} A_{\partial}^{3} A_{\partial}^{\prime}+1\right) \nabla^{\mu} \phi\right]=m_{\phi}^{2} \phi .
$$

A function of the form $A_{\partial}=e^{\beta_{\partial} K / 2}$ with $\beta_{\partial}<0$ would reverse the coefficient of the principal part of the partial differential equation on the left, which can also be seen in the linearized equation around $\phi=0$

$$
\left(-4 \pi \tilde{T} \beta_{\partial}+1\right) \square \phi=m_{\phi}^{2} \phi+4 \pi \beta_{\partial} \nabla_{\mu} \tilde{T} \nabla^{\mu} \phi .
$$

A scalar with the "wrong" kinetic term is called a ghost, and it also causes exponential growth. This phenomena is called "ghost-based spontaneous scalarization" [11. A physically relevant scalarization process would require the eventual suppression of the growth due to nonlinear terms and the stability of the final scalarized solution. The exponential form of $A_{\partial}$ suggests such a suppression, but the stability of these solutions is not yet known. The coefficient of $\square \phi$ is positive in the far field, and hence it has to vanish at some point near the scalarization region. Such differential equations can lead to problematic behavior, which requires detailed mathematical analysis [11].

This method of scalarizing neutron stars can also be adapted to more general gravity theories just like the extended spontaneous scalarization theories in (Eq. 4). 
Namely, the action

$$
\begin{aligned}
& \frac{1}{16 \pi} \int d^{4} x \sqrt{-g}\left[R-2 \nabla_{\mu} \phi \nabla^{\mu} \phi-2 m_{\phi}^{2} \phi^{2}\right] \\
+ & \left.\frac{1}{16 \pi} \int d^{4} x \sqrt{-g} \bar{\lambda}_{\partial}^{2} f_{\partial}(K) \mathcal{L}_{\chi}\left[\chi, g_{\mu \nu}\right]\right],
\end{aligned}
$$

with the equation of motion

$$
\nabla_{\mu}\left[\left(-\frac{\bar{\lambda}_{\partial}^{2}}{2} \mathcal{L}_{\chi} f_{\partial}^{\prime}(K)+1\right) \nabla^{\mu} \phi\right]=m_{\phi}^{2} \phi
$$

would also have a ghostlike instability and spontaneous growth for a choice such as $f_{\partial}=1-e^{\beta_{\partial} K}$ with appropriate $\beta_{\partial}$.

Despite the similarities between the original ghostbased spontaneous scalarization through matter coupling (Eq.16) and through generic coupling (Eq. 18), we need to take additional care with the latter. In Eq. 16, the additional terms that arise from the nonminimal coupling introduce at most second-derivative terms into the equations of motion. This is still the case for the derivatives of $\phi$ in Eq. 18, however, we also have $\nabla_{\mu} \mathcal{L}_{\chi}$. This term again brings at most second derivatives if $\mathcal{L}_{\chi}$ is the Maxwell Lagrangian as in the Einstein-Maxwell-scalar theories. On the other hand, if we couple to the GaussBonnet term, we now have $\nabla_{\mu} \mathcal{R}^{2}$ appearing in the equation of motion, which has three derivatives acting on the dynamical field $g_{\mu \nu}$. We have not closely examined the metric equation of motion (the modified Einstein equation) in our study so far, since it did not play a crucial role in the onset of the instability. However, it also contains third derivatives, this time of the scalar field. ${ }^{2}$

Aside from changing the principal part of the equations of motion, which is a major modification, more than two time derivatives are potential sources of unphysical behavior through Ostrogradsky's theorem [17]. This is one of the main reasons that makes the scalar coupling (without derivatives) to the Gauss-Bonnet term very important: it is the only higher-curvature term that provides a second-order partial differential equation as the equation of motion. This aspect of the theory is apparently lost once the coupling contains derivatives of the scalar. In fact, any derivative term has the same effect as we will shortly see.

Extra derivatives mean that extended spontaneous tensorization with derivative coupling should be analyzed with more care than the direct scalar or vector field coupling, but it can still be physically relevant. First, theories with more than two derivatives in their equation of motion can be viable due to symmetries which allow the expression of higher derivatives in terms of lower ones, which ultimately renders them second order, such

\footnotetext{
${ }^{2}$ Roughly, the variation of the Gauss-Bonnet term with respect to the metric moves its two derivatives to its coefficient $f_{\partial}$ through partial integration, resulting in three derivatives of $\phi$.
}

as $f(R)$ gravity theories and certain Beyond-Horndeski theories [18 20]. We could not identify such symmetries for Eq. 18, but they can be nontrivial to identify. Second, even though the Gauss-Bonnet term is unique in providing second-order equations in four dimensions, there have been countless studies on other higher curvature terms [2], including theories with extended spontaneous scalarization [15]. Such theories are investigated using the "effective field theory" approach where they are thought to be low-energy approximations to an unknown well-behaved theory [2]. Hence, the higher-derivative terms are treated as perturbations to understand the well-behaved theory, even though they form the principal part of the partial differential equations when taken at face value. Equations of motion arising from Eq. 17 can also be treated in this manner.

We can consider other fields with derivative couplings as well. For example, one can consider a theory of vector derivative coupling

$$
\begin{aligned}
& \frac{1}{16 \pi} \int d^{4} x \sqrt{-g}\left[R-F_{\mu \nu} F^{\mu \nu}-2 m_{X}^{2} X_{\mu} X^{\mu}\right] \\
+ & \left.\frac{1}{16 \pi} \int d^{4} x \sqrt{-g} \bar{\lambda}_{\eta}^{2} f_{\eta}(\eta) \mathcal{L}_{\chi}\left[\chi, g_{\mu \nu}\right]\right],
\end{aligned}
$$

with $\eta=F_{\mu \nu} F^{\mu \nu} / 2$, and equation of motion

$$
\nabla_{\rho}\left[\left(-\frac{\bar{\lambda}_{\eta}^{2}}{2} \mathcal{L}_{\chi} f_{\eta}^{\prime}(\eta)+1\right) F^{\rho \mu}\right]=m_{X}^{2} X^{\mu}
$$

A choice like $f_{\eta}=1-e^{\beta_{\eta} \eta / 2}$ would provide ghost-based spontaneous growth of the vector field. This theory also features higher derivatives in its equations of motion, and its physical nature should be understood with the same caveats as the scalar ghosts we discussed above.

\section{B. Spontaneous spinorization}

There are tachyon- and ghost-based spontaneous growth options for scalars and vectors, but the derivative coupling is a necessity in some cases which leads to ghostlike behavior. Let us try to continue the logic of Sec. III, and investigate how a spinor can spontaneously grow through the action

$$
\begin{aligned}
\frac{1}{16 \pi} \int d^{4} x \sqrt{-g}[ & R+\frac{1}{2}\left(\bar{\psi} \gamma^{\mu}\left(\nabla_{\mu} \psi\right)-\left(\nabla_{\mu} \bar{\psi}\right) \gamma^{\mu} \psi\right) \\
& \left.-m_{\psi} \bar{\psi} \psi+\bar{\lambda}_{\psi}^{2} f_{\psi} \mathcal{L}_{\chi}\left[\chi, g_{\mu \nu}\right]\right]
\end{aligned}
$$

where $\psi$ is a four-component Dirac bispinor with mass $m_{\psi}, \gamma^{\mu}$ are the curved-space Dirac gamma matrices that satisfy $\gamma^{(\mu} \gamma^{\nu)}=g^{\mu \nu}$, and $\bar{\psi} \equiv-i \psi^{\dagger} \hat{\gamma}^{0}$ where $\hat{\gamma}^{(\mu} \hat{\gamma}^{\nu)}=\eta^{\mu \nu}$ is the defining relationship of the flat spacetime gamma matrices. The spinor terms comprise what we saw in all of the theories in this study: the canonical action of the field that we want to grow. What should $f_{\psi}$ 
be so that the equation of motion implies an instability of $\psi=0$ ? The answer to this question is less transparent than its counterparts for scalars or vectors.

The terms that introduced the tachyonic $\left(\phi^{2}, X_{\mu} X^{\mu}\right)$ and ghostlike instabilities $(K, \eta)$ were all quadratic in nature for scalars and vectors, and we could reverse their signs to obtain an instability. The equation of motion for a spinor is first order, and reversing the sign of the derivative term or the mass term does not change its nature. Nevertheless, a solution has been found in the context of generalizing the DEF theory to spinors, which utilizes the multicomponent nature of $\psi$ 21]. The choice is $f_{\psi}=f_{\psi}\left(\mathcal{L}_{\psi}^{5, K}\right)$ where

$$
2 \mathcal{L}_{\psi}^{5, K}=\bar{\psi} \hat{\gamma}^{5} \gamma^{\mu}\left(\nabla_{\mu} \psi\right)-\left(\nabla_{\mu} \bar{\psi}\right) \hat{\gamma}^{5} \gamma^{\mu} \psi
$$

with

$$
\gamma^{5} \equiv \frac{i}{4 !} \epsilon_{\mu \nu \rho \sigma} \gamma^{\mu} \gamma^{\nu} \gamma^{\rho} \gamma^{\sigma}=\frac{i}{4 !} \tilde{\epsilon}_{a b c d} \hat{\gamma}^{a} \hat{\gamma}^{b} \hat{\gamma}^{c} \hat{\gamma}^{d}=\hat{\gamma}^{5},
$$

which is the flat-space gamma matrix.

We can see that $f_{\psi}\left(\mathcal{L}_{\psi}^{5, K}\right)$ provides an instability through the equation of motion

$$
\left(\zeta_{\psi} \hat{\gamma}^{5}+\mathbb{I}\right) \gamma^{\mu} \nabla_{\mu} \psi-\left[m_{\psi}-\frac{1}{2}\left(\nabla_{\mu} \zeta_{\psi}\right) \hat{\gamma}^{5} \gamma^{\mu}\right] \psi=0,
$$

or after some basic manipulation

$$
\gamma^{\mu} \nabla_{\mu} \psi-\frac{\mathbb{I}-\zeta_{\psi} \hat{\gamma}^{5}}{1-\zeta_{\psi}^{2}}\left[m_{\psi}-\frac{1}{2}\left(\nabla_{\mu} \zeta_{\psi}\right) \hat{\gamma}^{5} \gamma^{\mu}\right] \psi=0
$$

where

$$
\zeta_{\psi} \equiv \bar{\lambda}_{\psi}^{2} \mathcal{L}_{\chi} f_{\psi}^{\prime}\left(\mathcal{L}_{\psi}^{5, K}\right)
$$

Like the ghost-based spontaneous tensorization scenarios before, spontaneous spinorization also brings higher derivative terms. When $\mathcal{L}_{\chi}$ is a curvature term, the situation is similar to that of ghost-based spontaneous scalarization and vectorization, and we need to understand the theory as an effective one, unless there is a yet unidentified symmetry that cancels the higher derivatives.

When $\mathcal{L}_{\chi}$ is not a curvature term (e.g. EinsteinMaxwell-scalar theories) there is no higher-derivative problem, but the nature of the spinor equation seems to change drastically: $\zeta_{\psi}$ contains first derivatives of $\psi$, and $\nabla_{\mu} \zeta_{\psi}$ seems to contain second derivatives of $\psi$. Hence, Eq. 25 is a second-order partial differential equation in $\psi$ as opposed to the first-order formulation in GR. However, this is not actually the case because Eq. 25 implies

$$
\mathcal{L}_{\psi}^{5, K}=-m \frac{\zeta_{\psi}}{1-\zeta_{\psi}^{2}} \bar{\psi} \psi
$$

This, together with Eq. 26, is equivalent to

$$
\frac{\zeta_{\psi}}{\bar{\lambda}_{\psi}^{2} \mathcal{L}_{\chi}}=f_{\psi}^{\prime}\left(-m \bar{\psi} \psi \frac{\zeta_{\psi}}{1-\zeta_{\psi}^{2}}\right) \text {. }
$$

This implicit relationship can be used to express $\zeta_{\psi}$ as a function of $\bar{\psi} \psi$ and $\mathcal{L}_{\chi}$, but not their derivatives. The spinor equation of motion remains first order as a consequence.

Note that our initial aim was not a derivative coupling or a ghostlike instability. We were merely trying to imitate the transition from scalars to vectors, and finally to spinors, which naturally required a derivative coupling. Hence, the effort to extend spontaneous growth in terms of types of fields or instabilities, and the problems they bring with them, cannot always be separated. We should also add that a spinor that grows spontaneously cannot satisfy the occupation number rules of quantum mechanics, and hence it is strictly a classical object [21].

\section{Higgs-based spontaneous growth}

Another mechanism to generate spontaneous growth can be obtained by noting that the coupling that leads to the spontaneous growth in DEF and ESTGB theories acts as a mass generation mechanism, albeit with the wrong sign. There is already a well-known mass generation mechanism in physics, the Higgs mechanism. Hence, the Higgs mechanism can also be used to incite spontaneous growth by generating a mass-squared term with the wrong sign. This recipe has been applied to extend the DEF theory to a spontaneously growing vector field $X_{\mu}$ due to its coupling to a complex Higgs scalar $\Phi$ as in the action $[22]$

$$
\begin{aligned}
& \frac{1}{16 \pi} \int d^{4} x \sqrt{-g} R-\frac{1}{16 \pi} \int d^{4} x \sqrt{-g} F_{\mu \nu} F^{\mu \nu} \\
- & \frac{1}{16 \pi} \int d^{4} x \sqrt{-g}\left(2 \overline{D_{\mu} \Phi} D^{\mu} \Phi+2 V(\bar{\Phi} \Phi)\right) \\
+ & S_{m}\left[\psi_{m}, A_{H}^{2} g_{\mu \nu}\right],
\end{aligned}
$$

where $V(\bar{\Phi} \Phi)=m_{0}^{2}\left(u^{2}-\bar{\Phi} \Phi\right)^{2} /\left(2 u_{0}^{2}\right)$ is the Mexican-hat potential of the Higgs field which provides its nonzero expectation value, $D_{\mu} \Phi=\left(\nabla_{\mu}-i e X_{\mu}\right) \Phi$ is the gaugecovariant derivative with coupling constant $e$, and $A_{H}=$ $A_{H}\left(D^{2}\right)$ where $D^{2} \equiv \overline{D_{\mu} \Phi} D^{\mu} \Phi$ and an overbar denotes complex conjugation. The argument of $A_{H}$ is chosen in this way since the mass of $X_{\mu}$ is generated due to the $\bar{\Phi} \Phi X_{\mu} X^{\mu}$ term inside this expression. This means that an appropriate functional form of $A_{H}$ can invert the sign of the effective vector field mass.

The vector field equation of motion is

$$
\nabla^{\nu} F_{\nu \mu}=\left(-8 \pi \tilde{T} A_{H}^{3} A_{H}^{\prime}+1\right)\left(e^{2} \bar{\Phi} \Phi X_{\mu}+J_{\mu}^{\Phi}\right),
$$

where $J_{\mu}^{\Phi}=i e\left(\bar{\Phi} \nabla_{\mu} \Phi-\Phi \nabla_{\mu} \bar{\Phi}\right) / 2$. The typical choice of $A_{H}=e^{\beta_{H} D^{2} / 2}$ changes the sign of the effective mass of $X_{\mu}$, and leads to spontaneous growth as explained in detail in Ref. 22. 
This mechanism is extended to more generic couplings

$$
\begin{aligned}
& \frac{1}{16 \pi} \int d^{4} x \sqrt{-g}\left[R-F_{\mu \nu} F^{\mu \nu}-2 \overline{D_{\mu} \Phi} D^{\mu} \Phi-2 V(\bar{\Phi} \Phi)\right] \\
& \left.+\frac{1}{16 \pi} \int d^{4} x \sqrt{-g} f_{H} \mathcal{L}_{\chi}\left[\chi, g_{\mu \nu}\right]\right],
\end{aligned}
$$

where $f_{H}=f_{H}\left(D^{2}\right)$ with the typical example $f_{H}=1-$ $e^{\beta_{H} D^{2} / 2}$. The equation of motion is given by

$$
\left.\nabla^{\nu} F_{\nu \mu}=\left(-\frac{\mathcal{L}_{\chi}}{2} \widehat{f_{H}^{\prime}\left(D^{2}\right.}\right)+1\right)\left(e^{2} \bar{\Phi} \Phi X_{\mu}+J_{\mu}^{\Phi}\right)
$$

The relationship between the Higgs mechanism and spontaneous growth was first noted in the opposite direction, where spontaneous growth is used instead of the potential $V(\bar{\Phi} \Phi)$ to provide a nonzero expectation value for $\Phi$, which is called the gravitational Higgs mechanism 23. It is possible to have a second form of Higgsbased spontaneous growth through this mechanism (see the discussion around Eq. 26 in Ref. 222]). Both the gravitational Higgs mechanism and the Higgs-based spontaneous growth mechanism inspired by it can also be adapted to generic couplings, as in Eq. 31 .

The Higgs mechanism can also provide the mass term for non-Abelian gauge bosons, which is critical for their quantum theory. Consequently, one can also spontaneously grow such fields $W_{\mu}^{a}$ through the Higgs mechanism as well in a theory with the action 22 .

$$
\begin{gathered}
\frac{1}{16 \pi} \int d^{4} x \sqrt{-g}\left[R-F^{a \mu \nu} F_{\mu \nu}^{a}-2\left(D_{\mu} \Phi\right)^{\dagger} D^{\mu} \Phi\right. \\
\left.-2 V\left(\Phi^{\dagger} \Phi\right) f_{Y M} \mathcal{L}_{\chi}\left[\chi, g_{\mu \nu}\right]\right],
\end{gathered}
$$

where

$$
\begin{aligned}
F_{\mu \nu}^{a} & =\nabla_{\mu} W_{\nu}^{a}-\nabla_{\nu} W_{\mu}^{a}+e f^{a b c} W_{\mu}^{b} W_{\nu}^{c} \\
D_{\mu} \Phi & =\nabla_{\mu} \Phi-i e W_{\mu}^{b} T^{b} \Phi
\end{aligned}
$$

where $a, b, c$ label the generators of the Lie algebra of the gauge group $\left(T^{a}\right), f^{a b c}$ are the structure constants and $\dagger$ indicates Hermitian conjugation. The natural choice for spontaneous growth is $f_{Y M}=1-e^{\beta_{Y M}\left(D_{\mu} \Phi\right)^{\dagger} D^{\mu} \Phi / 2}$.

The Higgs mechanism generates mass, and hence it can be considered to be closely associated with a tachyonic instability. This is indeed the case for the vector field in Eq. 32 but the coupling terms $\overline{D_{\mu} \Phi} D^{\mu} \Phi$ and $\left(D_{\mu} \Phi\right)^{\dagger} D^{\mu} \Phi$ also contain derivative expressions for the Higgs field itself. This means that even though Eq. 32 is free of the higher-derivative problems we encountered for ghost-based scalarization and vectorization, the equation of motion for $\Phi$ contains third-order derivatives of $g_{\mu \nu}$, and the modified Einstein equation for $g_{\mu \nu}$ contains third-order derivatives of $\Phi$. Our previous discussion about the physical meaning of derivative couplings applies to all Higgs-based spontaneous growth theories as well.

\section{DISCUSSION}

This study aimed to combine two approaches to gravity theories with hairy compact object solutions: generalizing the scalar-dependent couplings of the action from matter to more general terms, as in the ESTGB theory, and generalizing the field that goes through spontaneous growth beyond the scalar, as in spontaneous tensorization. The new family of extended theories of spontaneous growth has the general action

$$
\frac{1}{16 \pi} \int d^{4} x \sqrt{-g}\left[R+\mathcal{L}_{\xi}+f_{\xi}(\xi, \partial \xi) \mathcal{L}_{\chi}\left[\chi, g_{\mu \nu}\right]\right],
$$

where $\xi$ is the field that spontaneously grows whose action is $\mathcal{L}_{\xi}$, and $\mathcal{L}_{\chi}$ is the Lagrangian that drives the spontaneous growth which can be purely curvature (e.g., ESTGB), or may also depend on other fields $\chi$ (e.g., Einstein-Maxwell-scalar).

The extended family has considerably richer phenomenology compared to spontaneous scalarization. Black holes can also be scalarized, which is not possible in spontaneous tensorization. In theories where the coupling is to a term that contains another field $\chi$, the phenomenon goes beyond gravity, since such terms can be nonzero even in flat spacetime and cause growth, as in Einstein-Maxwell-scalar theories. The far-field behavior of a curvature term such as the Gauss-Bonnet term is radically different from that of neutron star matter which only lives in a finite region of space. This means that the weak-field behavior of extended spontaneous tensorization theories is also more varied compared to spontaneous tensorization, which may provide further observable signatures.

We constructed these theories in a way that avoids higher derivative terms of $\xi$ so that there are no obvious runaway instabilities. However, when the coupling $f_{\xi}$ is to a curvature term, we saw that even a single derivative of $\xi$ leads to more than two time derivatives in the equations of motion. Such theories can be examined under the umbrella of "effective theories". An alternative, more conservative, approach is to disregard any higher derivative theory, using our results as "no-go" theorems to explore the limits of spontaneous growth phenomena. This latter path is also interesting since the original idea of spontaneous tensorization applied to matter couplings does not suffer from higher derivatives. This means, theories where the field couples to different Lagrangians $\left(\mathcal{L}_{\chi}\right)$ can be radically different. Understanding the reasons for this can provide further insights into nature of gravity in general.

When the coupling function $f_{\xi}$ does not have derivative dependence, there is no higher-derivative problem, as in the extended spontaneous vectorization in Eq. 12 . However, this is not a complete guarantee of well-posedness. To start with, there is already an instability in these theories to incite the spontaneous growth, which is known to be shut off in the DEF theory due to nonlinear effects as the field grows [16. This logic can also be 
continued to extended scalar-tensor theories [12 14, but the mechanisms that regularize the instabilities have not been closely investigated beyond these, and their effectiveness in the nonlinear regime is not definitely known. Ghost-like instabilities need special care since some of their solutions in DEF-like theories are known to show unusual features in astrophysical systems even when they do not lead to higher-derivative equations of motion [1]. It might be that there are more stringent requirements on $f_{\xi}$ than the examples we mentioned here $\left(1-e^{\beta_{\xi} \cdots}\right)$, which were based on the analysis of the linearized equations of motion and common practices in the DEF theory [16].

We should mention that the exact form of Eq. 35 does not exhaust all cases of spontaneous growth. Many known examples are closely related to this form such as axionic instabilities 24, spontaneous growth of hidden vector fields [25], and the extended massive gravity theories where the coupling between the two metrics is a function of a dynamical scalar 26. However, one can look for even more general actions, for example, by giving up our adopted condition that the action of the spontaneously growing field is the canonical one in the Einstein frame. For the case of scalars, all possible action terms that lead to second-order-in-time equations have been classified as Horndeski [27] and beyond-Horndeski [28] theories. These may be starting points for more general forms of spontaneous growth, and this strategy can also be repeated for vectors as well.

The spontaneous growth mechanism in Eq. 35 can be adapted to other cases (such as more than one field) in a relatively straightforward manner. However, it has certain limits which are similar to those of spontaneous tensorization theories with matter coupling. For example, it does not seem to be possible to find a theory that would feature spontaneously growing spin-2 fields with local Lorentz symmetry, since any coupling between two spin2 fields other than that of de Rham-Gabadadze-Tolley massive gravity would contain ghosts [11, 29].

The relationship between extended spontaneous tensorization theories and their nonextended counterparts is not merely a mathematical similarity, these theories share observable signatures. First, their deviation from GR is nonperturbativ (i.e. order-of-unity) in the strong field regime. This makes them a relatively easy target for observatories, and theoretical predictions about one theory in the family can provide valuable insight for the group as a whole. Second, the appeal of the DEF theory was the fact that it avoided the constraints on the weak field that disfavored other scalar-tensor theories such as that of Brans-Dicke theory [3]. This feature is also shared by spontaneous tensorization, both extended or nonextended. Other modes of field growth such as dynamical scalarization in the DEF theory [30] are also likely to have analogs in all extended spontaneous growth theories.

\section{Acknowledgments}

We thank Thomas Sotiriou for valuable discussions on the content of the paper and on spontaneous growth phenomena in general. The author is supported by Grant No. 117F295 of the Scientific and Technological Research Council of Turkey (TÜBITAK). We would like to acknowledge networking and travel support by the European Cooperation in Science and Technology (COST) Action CA16104: GWverse.
[1] E. Berti et al., Class. Quant. Grav. 32, 243001 (2015), 1501.07274 .

[2] L. Barack et al. (2018), 1806.05195.

[3] T. Damour and G. Esposito-Farèse, Phys. Rev. Lett. 70, 2220 (1993), URL http://link.aps.org/doi/10.1103/ PhysRevLett.70.2220

[4] C. M. Will, Living Rev. Relativity 4 (2001), http://www. livingreviews.org/lrr-2001-4, arXiv:gr-qc/0103036.

[5] B. P. Abbott et al. (LIGO Scientific and Virgo Collaborations) (2018), 1811.12907.

[6] H. O. Silva, J. Sakstein, L. Gualtieri, T. P. Sotiriou, and E. Berti, Phys. Rev. Lett. 120, 131104 (2018), 1711.02080.

[7] D. D. Doneva and S. S. Yazadjiev, Phys. Rev. Lett. 120, 131103 (2018), 1711.01187.

[8] G. Antoniou, A. Bakopoulos, and P. Kanti, Phys. Rev. Lett. 120, 131102 (2018), 1711.03390.

[9] C. A. R. Herdeiro, E. Radu, N. Sanchis-Gual, and J. A. Font, Phys. Rev. Lett. 121, 101102 (2018), 1806.05190.

[10] F. M. Ramazanoğlu, Phys. Rev. D96, 064009 (2017), 1706.01056.

[11] F. M. Ramazanoğlu, Phys. Rev. D97, 024008 (2018), 1710.00863 .
[12] J. L. Blázquez-Salcedo, D. D. Doneva, J. Kunz, and S. S. Yazadjiev, Phys. Rev. D98, 084011 (2018), 1805.05755.

[13] M. Minamitsuji and T. Ikeda (2018), 1812.03551.

[14] H. O. Silva, C. F. B. Macedo, T. P. Sotiriou, L. Gualtieri, J. Sakstein, and E. Berti (2018), 1812.05590.

[15] Y.-X. Gao, Y. Huang, and D.-J. Liu (2018), 1808.01433.

[16] F. M. Ramazanoğlu and F. Pretorius, Phys. Rev. D93, 064005 (2016), 1601.07475.

[17] R. P. Woodard, Scholarpedia 10, 32243 (2015), revision \#186559.

[18] T. P. Sotiriou and V. Faraoni, Rev. Mod. Phys. 82, 451 (2010), 0805.1726.

[19] M. Zumalacárregui and J. Garcia-Bellido, Phys. Rev. D89, 064046 (2014), 1308.4685.

[20] F. M. Ramazanoğlu, Phys. Rev. D99, 044003 (2019), 1901.00194 .

[21] F. M. Ramazanoğlu, Phys. Rev. D98, 044011 (2018), 1804.00594.

[22] F. M. Ramazanoğlu, Phys. Rev. D98, 044013 (2018), 1804.03158.

[23] A. Coates, M. W. Horbatsch, and T. P. Sotiriou, Phys. Rev. D95, 084003 (2017), 1606.03981.

[24] M. Boskovic, R. Brito, V. Cardoso, T. Ikeda, and 
H. Witek (2018), 1811.04945.

[25] L. Annulli, V. Cardoso, and L. Gualtieri (2019), 1901.02461.

[26] J. Zhang and S.-Y. Zhou, Phys. Rev. D97, 081501 (2018), 1709.07503.

[27] G. W. Horndeski, International Journal of Theoretical Physics 10, 363 (1974), ISSN 1572-9575, URL https: //doi.org/10.1007/BF01807638
[28] J. Gleyzes, D. Langlois, F. Piazza, and F. Vernizzi, Phys. Rev. Lett. 114, 211101 (2015), URL https://link. aps. org/doi/10.1103/PhysRevLett.114.211101

[29] C. de Rham, Living Rev. Rel. 17, 7 (2014), 1401.4173.

[30] E. Barausse, C. Palenzuela, M. Ponce, and L. Lehner, Phys. Rev. D 87, 081506 (2013), 1212.5053. 